\title{
Effects of Variable Thermal Conductivity and Heat Source / Sink on MHD Flow Near a Stagnation Point on a Linearly Stretching Sheet
}

\author{
P. R. Sharma ${ }^{1}$ and G. Singh ${ }^{2}$ \\ ${ }^{1}$ Department of Mathematics, University of Rajasthan, Jaipur - 302004, India \\ ${ }^{2}$ Birla Institute of Technology (Jaipur Ext.),27,Malviya Industrial Area, Jaipur-302017,India \\ Email:profprsharma@yahoo.com,garry_mal@yahoo.com
}

(Received September 30, 2007; accepted April 20, 2008)

\begin{abstract}
Aim of the paper is to investigate effects of variable thermal conductivity and heat source/sink on flow of a viscous incompressible electrically conducting fluid in the presence of uniform transverse magnetic field and variable free stream near a stagnation point on a non-conducting stretching sheet. The equations of continuity, momentum and energy are transformed into ordinary differential equations and solved numerically using shooting method. The velocity and temperature distributions are discussed numerically and presented through graphs. Skin-friction coefficient and the Nusselt number at the sheet are derived, discussed numerically and their numerical values for various values of physical parameter are presented through Tables.
\end{abstract}

Keywords: Steady, boundary layer, MHD, variable thermal conductivity, stagnation point, source/sink, stretching sheet, skin-friction coefficient and Nusselt number.

\section{NOMENCLATURE}

$b \quad$ free stream velocity parameter

$B_{0} \quad$ magnetic field intensity

$C \quad$ stretching sheet parameter

$C_{f} \quad$ skin-friction coefficient

$C_{p} \quad$ specific heat at constant pressure

$M \quad$ Hartmann number $\left\{=\left(\sigma B_{o}{ }^{2} / \rho c\right)^{1 / 2}\right\}$

$\mathrm{Nu} \quad$ Nusselt number

$\operatorname{Pr} \quad$ Prandtl number $\left(=\mu C_{p} / \kappa\right)$

$\mathrm{q}_{\mathrm{w}} \quad$ rate of heat transfer

Q volumetric rate of heat generation/absorption heat source/sink parameter $\left\{=Q / \rho C_{p} c\right\}$

fluid temperature

$\begin{array}{ll}T_{\infty} & \text { free stream temperature } \\ T_{w} & \text { temperature of stretching sheet }\end{array}$

$u, v \quad$ velocity components along $\mathrm{x}$ - and $\mathrm{y}$ - axes, respectively

$u_{w}(x) \quad$ velocity of stretching sheet

$U(x) \quad$ free stream velocity $(=b x)$

$x, y \quad$ Cartesian coordinates along $\mathrm{x}-, \mathrm{y}$ - axes, respectively

\section{INTRODUCTION}

Study of heat transfer in boundary layer over stretching surface find applications in extrusion of plastic sheets, polymer, spinning of fibers, cooling of elastic sheets etc.

\begin{tabular}{|c|c|}
\hline \\
\hline \multicolumn{2}{|c|}{$\varepsilon \quad$ perturbation parameter } \\
\hline$\eta$ & similarity parameter $\left\{=(c / v)^{1 / 2} y\right\}$ \\
\hline$\eta_{\infty}$ & $\begin{array}{l}\text { value of } \eta \text { at which boundary conditions is } \\
\text { achieved }\end{array}$ \\
\hline$\kappa$ & uniform thermal conductivity \\
\hline$\kappa^{*}$ & variable thermal conductivity \\
\hline$\lambda$ & $\begin{array}{l}\text { ratio of free stream velocity parameter to } \\
\text { stretching sheet parameter }\{=b / c\}\end{array}$ \\
\hline$\mu$ & coefficient of viscosity \\
\hline$v$ & kinematic viscosity \\
\hline$\rho$ & density of fluid \\
\hline$\psi$ & stream function \\
\hline$\sigma$ & electrical conductivity \\
\hline$\theta$ & dimensionless temperature \\
\hline & $\left\{=\left(T-T_{\infty}\right) /\left(T_{w}-T_{\infty}\right)\right\}$ \\
\hline$\tau_{w}$ & shear stress \\
\hline
\end{tabular}

The quality of final product depends on the rate of heat transfer and therefore cooling procedure has to be controlled effectively. The MHD flow in electrically conducting fluid can control the rate of cooling and the 
desired quality of product can be achieved [Chakrabarti and Gupta (1979)]. Liquid metals have small Prandtl number of order 0.01 0.1(e.g. $\operatorname{Pr}=0.01$ is for Bismuth, $\operatorname{Pr}=0.023$ for mercury etc.) and are generally used as coolants because of very large thermal conductivity.

Flow in the neighborhood of a stagnation point in a plane was initiated by Hiemenz (1911). Stagnation point flows have been discussed by Pai (1956), Schlichting (1968) and Bansal (1977) etc. Kay (1966) reported that thermal conductivity of liquids with low Prandtl number varies linearly with temperature in range of $0^{\circ} \mathrm{F}$ to $400^{\circ} \mathrm{F}$. Crane (1970) presented the flow over a stretching sheet and obtained similarity solution in closed analytical form. Arunachalam and Rajappa (1978) considered forced convection in liquid metals (fluid with low Prandtl number) with variable thermal conductivity and capacity in potential flow and derived explicit closed form of analytical solution. Fluid flow and heat transfer characteristics on stretching sheet with variable temperature condition have been investigated by Grubka and Bobba (1985). Watanabe (1986, 1988) discussed stability of boundary layer and effect of suction/injection in MHD flow under pressure gradient. Noor (1992) studied the characteristics of heat transfer on stretching sheet. Convective heat transfer at a stretching sheet has been presented by Vajravelu and Nayfeh (1993). Sharma and Jat (1994) analysed flow and heat transfer between two vertical plates under viscous fluid injection through porous plate, the other being a stretching sheet. Chiam (1997) discussed the heat transfer in fluid flow on stretching sheet at stagnation point in presence of internal dissipation, heat source/sink and stress with constant fluid properties. Chen (1998) considered laminar mixed convection adjacent to vertical, continuously stretching sheet. Chaim (1998) studied heat transfer in fluid flow of low Prandtl number with variable thermal conductivity, induced due to stretching sheet and compared the numerical results with perturbation solution. Chamka and Khaled (2000) considered Hiemenz flow in the presence of magnetic field through porous media. Sharma and Mishra (2001) investigated steady MHD flow through horizontal channel: lower being a stretching sheet and upper being a permeable plate bounded by porous medium. Sriramalu et.al. (2001) studied steady flow and heat transfer of a viscous incompressible fluid flow through porous medium over a stretching sheet. Mahapatra and Gupta (2001) investigated the magnetohydrodynamic stagnation-point flow towards isothermal stretching sheet and pointed that velocity decreases/increases with the increase in magnetic field intensity when free stream velocity is smaller/greater, repectively than the stretching velocity. Mahapatra and Gupta (2002) studied heat transfer in stagnation-point flow towards stretching sheet with viscous dissipation effect. Khan et.al. (2003) presented viscoelastic MHD flow, heat and mass transfer over a porous stretching sheet with dissipation energy and stress work. Pop et.al. (2004) discussed the flow over stretching sheet near a stagnation point taking radiation effect. Seddeek and Salem (2005) investigated the heat and mass transfer distributions on stretching surface with variable viscosity and thermal diffusivity.
Aim of the present paper is to investigate effects of variable thermal conductivity, heat source/sink and variable free stream on flow of a viscous incompressible electrically conducting fluid and heat transfer on a non-conducting stretching sheet in the presence of transverse magnetic field near a stagnation point. Linear stretching of the sheet is considered because of its simplicity in modelling of the flow and heat transfer over stretching surface and further it permits the similarity solution, which are useful in understanding the interaction of flow field with temperature field. The heat source and sink is included in the work to understand the effect of internal heat generation and absorption [Chaim (1998)].

\section{Formulation of The Problem}

Consider steady two-dimensional flow of a viscous incompressible electrically conducting fluid of variable thermal conductivity in the vicinity of a stagnation point on a non-conducting stretching sheet in the presence of transverse magnetic field and volumetric rate of heat generation/absorption. The stretching sheet has uniform temperature $T_{w}$, linear velocity $u_{w}(x)$. It is assumed that external field is zero, the electric field owing to polarization of charges and Hall effect are neglected. Stretching sheet is placed in the plane $y=0$ and $x$-axis is taken along the sheet as shown in the Fig. 1. The fluid occupies the upper half plane i.e. $y>0$.

The governing equations of continuity, momentum and energy under the influence of externally imposed transverse magnetic field [Bansal (1994)] with variable thermal conductivity in the boundary layer are

$$
\begin{aligned}
& \frac{\partial u}{\partial x}+\frac{\partial v}{\partial y}=0 \\
& u \frac{\partial u}{\partial x}+v \frac{\partial u}{\partial y}=-\frac{1}{\rho} \frac{\partial p}{\partial x}+v \frac{\partial^{2} u}{\partial y^{2}}-\frac{\sigma B_{0}^{2}}{\rho} u \\
& \rho C_{p}\left(u \frac{\partial T}{\partial x}+v \frac{\partial T}{\partial y}\right)=\frac{\partial}{\partial y}\left(\kappa^{*} \frac{\partial T}{\partial y}\right)+Q\left(T-T_{\infty}\right)
\end{aligned}
$$

The second derivatives of $u$ and $T$ w.r.t $x$ have been eliminated on the basis of magnitude analysis considering that Reynolds number is high. Hence the Navier-Stokes equation modifies into Prandtl's boundary layer equation.

In the free stream $u=U(x)=b x$, the equation (2) reduces to

$U \frac{d U}{d x}=-\frac{1}{\rho} \frac{\partial p}{\partial x}-\frac{\sigma B_{0}^{2}}{\rho} U$.

Eliminating $\frac{\partial p}{\partial x}$ between the equations (2) and (4), we obtain

$u \frac{\partial u}{\partial x}+v \frac{\partial u}{\partial y}=U \frac{d U}{d x}+v \frac{\partial^{2} u}{\partial y^{2}}-\frac{\sigma B_{0}^{2}}{\rho}(u-U)$.

The boundary conditions are 
$y=0: \quad u=u_{w}(x)=c x, v=0, T=T_{w}$,

$y \rightarrow \infty: u=U(x)=b x, T=T_{\infty}$.

Following Arunachalam and Rajappa (1978) and Chaim (1998), the thermal conductivity $\kappa^{*}$ is taken of form as given below

$$
\kappa^{*}=\kappa(1+\varepsilon \theta) \text {. }
$$

\section{METHOD OF SOLUTION}

Introducing the stream function $\psi(x, y)$ as defined by

$u=\frac{\partial \psi}{\partial y}$ and $v=-\frac{\partial \psi}{\partial x}$,

the similarity variable $\eta=(c / v)^{1 / 2} y$ and

$\psi(x, y)=(c v)^{1 / 2} x f(\eta)$,

into the equations (3) and (5), we get

$f^{\prime \prime \prime}+f f^{\prime \prime}-\left(f^{\prime}\right)^{2}-M^{2}\left(f^{\prime}-\lambda\right)+\lambda^{2}=0$,

and

$(1+\varepsilon \theta) \theta^{\prime \prime}+\varepsilon\left(\theta^{\prime}\right)^{2}+\operatorname{Pr} \theta^{\prime} f+\operatorname{Pr} S \theta=0$.

It is noted that equation (1) is identically satisfied. The corresponding boundary conditions are reduced to

$f(0)=0, f^{\prime}(0)=1, \theta(0)=1, f^{\prime}(\infty)=\lambda$ and $\theta(\infty)=0$.

The governing boundary layer and thermal boundary layer equations (10) and (11) with the boundary conditions (12) are solved using Runge-Kutta fourth order technique along with shooting method [Conte and Boor (1981)]. First of all, higher order non-linear differential equations (10) and (11) are converted into simultaneous linear differential equations of order first and they are further transformed into initial value problem applying the shooting technique. Once the problem is reduced to initial value problem, then it is solved using Runge-Kutta fourth order technique [Jain (1984), Jain, Iyengar and Jain (1985), Krishnamurthy and Sen (1986)].

\section{SKIN-FRICTION}

Skin-friction coefficient at the sheet is given by

$$
C_{f}=\frac{\tau_{w}}{\rho c(c v)^{1 / 2}}=x f^{\prime \prime}(0) \text {, }
$$

where $\tau_{w}\left\{=\mu\left(\frac{\partial u}{\partial y}+\frac{\partial v}{\partial x}\right)_{y=0}\right\}$ is the shear stress at the sheet.

\section{NUSSELT NUMBER}

The rate of heat transfer in terms of the Nusselt number at the sheet is given by

$$
N u=\left(\frac{v}{c}\right)^{1 / 2} \frac{q_{w}}{\kappa^{*}\left(T_{w}-T_{\infty}\right)}=-\theta^{\prime}(0),
$$

where $q_{w}=-\kappa^{*}\left(\frac{\partial T}{\partial y}\right)_{y=0}$.

\section{Particular Cases}

(i) In the absence of magnetic field i.e $M=0$, the results of the present paper are reduced to those obtained by Pop, Grosan, Pop (2004) and Mahapatra and Gupta (2002).

(ii) In the absence of magnetic field, heat source/sink and the free stream i.e. $M=0, S=0$ and $\mathrm{b}=0$, respectively; the results of the present paper are reduced to those obtained by Chiam (1998) for impermeable sheet.

(iii) In the absence of magnetic field, heat source/sink and constant thermal conductivity i.e. $M=0, S=0$ and $\varepsilon=0$, the results of the present paper are reduced to those obtained by Pop, Grosan, Pop (2004) in the absence of radiation effect with constant thermal conductivity and Mahapatra and Gupta (2002) in absence of viscous dissipation and constant thermal conductivity.

\section{RESULTS AND Discussion}

Equations (10) and (11) are solved using Runge-Kutta fourth order method for different values of $M, \lambda, \varepsilon, S$ and $\operatorname{Pr}$ taking step size 0.05 . While numerical simulation, step size $0.01,0.02$ and 0.03 were all checked and values of $f^{\prime \prime}(0)$ and $\theta^{\prime}(0)$ were found in each case correct up to five decimal places. Hence the scheme used in this paper stable and accurate.

It is observed from Table 1 that the numerical values of $f^{\prime \prime}(0)$ in the present paper when $M=0$ are in good agreement with results obtained by Pop, Grosan and Pop (2004) and Mahapatra and Gupta (2002). Former have used Runge-Kutta fourth order method and shooting technique, while the later have used finite difference technique and Thomas algorithm. The scheme exploited in the present paper is Runge Kutta fourth order method along with shooting technique. It is noted from Table 2 that the numerical values of $-\theta^{\prime}(0)$ in the present paper when $M=0, \lambda=0, \quad S=0$ and $\operatorname{Pr}=0.023$ are in good agreement with the results obtained by Chiam (1998).

It is seen from Table 3 that the numerical values of $-\theta^{\prime}(0)$ in the present paper when $M=0, S=0$ and $\varepsilon=0$ and $\operatorname{Pr}=0.05$ are in agreement with those obtained by Pop, Grosan, Pop (2004) and Mahapatra and Gupta (2002).

The skin-friction coefficient and Nusselt number are presented by equations (13) and (14) and are directly proportional $f^{\prime \prime}(0)$ and $-\theta^{\prime}(0)$, respectively. The effects of $M, \varepsilon, \lambda$ and $S$ on $f^{\prime \prime}(0)$ and $\theta^{\prime}(0)$ have been presented through Table 4 and Table 5, receptively.

Figure 2 shows that the boundary layer thickness decreases considerably as $\lambda$ increases which is shown with the help of dotted vertical lines at the points where $f^{\prime}(\eta)$ reaches the boundary condition. The increase in the value of $\lambda$ implies 
that free stream velocity increases in comparison to stretching velocity, which results in the increase in pressure and straining motion near stagnation point and hence thinning of boundary layer takes place. The phenomenon of thinning of boundary layer thus implies increased shear stress at the sheet, which is seen in Table 4 when $M=0$. It is important to note for $\lambda=1$, that there is no formation of boundary layer because the sheet velocity is equal to free stream velocity.

The Hartmann number represents the importance of magnetic field on the flow. The presence of transverse magnetic field sets in Lorentz force, which results in retarding force on the velocity field and therefore as Hartmann number increases, so does the retarding force and hence the velocity profiles decrease. This is shown in Fig. 3 when $\lambda<1$. In case when $\lambda>1$, which is just opposite to $\lambda<1$, as expected that the velocity profiles increase with the increase in the Hartmann number (Fig. 4). The direct implication of the above discussion, explains the results presented in Table 4, is that the shear stress at the sheet decreases due to increase in the Hartmann number when $\lambda<1$, while it increases with the increase in the Hartmann number when $\lambda>1$ [Mahapatra and Gupta (2001)].

It is noted from Table 5 that the rate of heat transfer at the sheet increases due to increase in the Prandtl number in the absence of magnetic field when thermal conductivity of the fluid is constant or variable in the presence/absence of the source/sink parameter. This is attributed to the fact that the thermal boundary layer for low Prandtl number fluid is thick and consequently the temperature gradient decreases with the decrease in Prandtl number. The rate of heat transfer at the sheet decreases due to increase in the Hartmann number when $\lambda=0.1$ or 0.5 , while the rate of heat transfer increases due to increase of the Hartmann number when $\lambda=2$; this is because of the magnetic field modifies the fluid velocity profile. The rate of heat transfer at the sheet increases due to increase in $\lambda$, which is due to the fact that thermal boundary thickness decreases due to increase in $\lambda$ and is also depicted in Fig. 10.

Figures 5 and 6 show that with the increase in the value of $\varepsilon$, temperature profile increases hence considering the thermal conductivity constant would lead to lower approximation of the temperature profile. It is observed from Figs. 7,8 and 9 that fluid temperature increases due to increase in the volumetric rate of heat generation, while it decreases in the case of volumetric rate of heat absorption for $\lambda=0.1,0.5$ and 2.0. It is also noted that the effect of volumetric rate of heat generation/absorption diminishes with the increase in $\lambda$, hence it can be concluded that with the decrease in boundary layer thickness, the effect of volumetric rate of heat generation/absorption on temperature profile also decreases in the case of low Prandtl number.

It is seen from Fig. 10 that fluid temperature decreases due to increase in $\lambda$. It is observed from Fig.11 that with the increase in Prandtl number, temperature profile decreases. This is because of the fact that with the increase in Prandtl number, thermal boundary layer thickness reduces. The effect is even more pronounced for small Prandtl number because the thermal boundary layer thickness is comparatively large. Figure 12 shows the effect of the Hartmann number on temperature profile for $\lambda=0.5$, which increases with the increase in the Hartmann number because the magnetic field retards the velocity of fluid and therefore temperature of fluid near the sheet is higher.

\section{CONCLUSIONS}

(i) Fluid velocity decreases due to increase in the Hartmann number for $\lambda<1$, while reverse effect is observed when $\lambda>1$. There is no boundary layer formation when $\lambda=1$.

(ii) Boundary layer thickness decreases due to increase in $\lambda$.

(iii) Fluid temperature increases due to increase in the thermal conductivity parameter, the Hartmann number or volumetric rate of heat source parameter, while it decreases due to increase in the Prandtl number, $\lambda$ or volumetric rate of sink parameter.

(iv) Shear stress at the sheet increases due to increase in $\lambda$, while it decreases due to increase in the Hartmann number when $\lambda<1$, but opposite behaviour is noted when $\lambda>1$.

(v) Rate of heat transfer at the sheet increases due to increase in the thermal conductivity parameter, while it decreases due to increase in $\lambda$ in absence of magnetic field and volumetric rate of heat source/sink parameter.

(vi) Rate of heat transfer at the sheet increases due to increase in the Prandtl number or $\lambda$, while it decreases due to increase in the Hartmann number.

\section{ACKNOWLEDGEMENTS}

The authors are thankful to the referee for his valuable suggestions

\section{REFERENCES}

Arunachalam, M. and N.R. Rajappa (1978). Forced convection in liquid metals with variable thermal conductivity and capacity. Acta Mechanica 31, 25-31.

Bansal, J.L. (1977). Viscous Fluid Dynamics. Oxford \& IBH Pub. Co., New Delhi.

Bansal, J.L. (1994). Magnetofluiddynamics of Viscous Fluids. Jaipur Pub. House, Jaipur, India.

Chamka, A.J. and A.R.A. Khaled (2000). Similarity solution for hydromagnetic mixed convection and mass transfer for Hiemenz flow though porous media. Int. Journal of Numerical Methods for Heat and Fluid Flow 10, 94-115. 
P. R. Sharma and G. Singh / JAFM, Vol. 2, No. 1, pp. 13-21, 2009.

Chakrabarti, A. and A.S. Gupta (1979). Hydromagnetic flow and heat transfer over a stretching sheet. Quarterly Journal of Applied Mathematics 37, 73-78.

Chen, C.H. (1998). Laminar mixed convection adjacent to vertical, continuously stretching sheet. Heat and Mass Transfer 33, 471-476.

Chiam, T.C. (1997). Magnetohydrodynamic heat transfer over a non-isothermal stretching sheet. Acta Mechanica 122, 169-179.

Chaim, T.C. (1998). Heat transfer in a fluid with variable thermal conductivity over stretching sheet. Acta Mechanica 129, 63-72.

Conte, S.D. and C. Boor (1981). Elementary Numerical Analysis, McGraw-Hill Book Co., New York.

Crane, L.J. (1970). Flow past a stretching plate. ZAMP 21, 645-647.

Grubka, L.J. and K.M. Bobba (1985). Heat transfer characteristics of a continuously stretching surface with variable temperature. Transactions of ASME Journal of Heat and Mass Transfer 107, 248-250.

Hiemenz, K. (1911). Die grenzschicht an einem in den gleich formigen flussigkeitsstrom eingetauchten geraden kreiszlinder. Dingl. Polytec. J. 326, 321-328.

Jain, M.K. (1984). Numerical Solution of Differential Equations, Wiley Eastern Ltd., New Delhi, India.

Jain, M.K., S.R. Iyengar, and R.K. Jain (1985). Numerical Methods for Scientific and Engineering Computation. Wiley Eastern Ltd., New Delhi, India.

Kay, W.M. (1966). Convective Heat and Mass Transfer, McGraw-Hill Book Co., New York.

Khan, S.K., A.M. Subhas, and R.M. Sonth (2003). Viscoelastic MHD flow, heat and mass transfer over a porous stretching sheet with dissipation energy and stress work. Heat and Mass Transfer 40, 47-57.

Krishnamurthy, E.V. and S.K. Sen (1986). Numerical Algorithms, Affiliated East-West Press Pvt. Ltd., New Delhi, India.

Mahapatra, T.R. and A.S. Gupta (2001). Magnetohydrodynamic stagnation-point flow towards a stretching sheet. Acta Mechanica 152, 191-196.

Mahapatra, T.R. and A.S. Gupta (2002). Heat transfer in stagnation-point flow towards a stretching sheet. Heat and Mass Transfer 38, 517-521.

Noor, A. (1992). Heat transfer from a stretching sheet. Int. J. Heat and Mass Transfer 4, 1128-1131.
Pai, S.I. (1956). Viscous Flow Theory I: Laminar Flow, D.Van Nostrand Co., New York.

Pop, S.R., T. Grosan and I. Pop (2004). Radiation effect on the flow near the stagnation point of a stretching sheet. Technische Mechanik 25, 100-106.

Schlichting, H. (1968). Boundary Layer Theory. McGrawHill Book Co., New York.

Seddeek, M.A. and A.M. Salem (2005). Laminar mixed convection adjacent to vertical continuously stretching sheet with variable viscosity and variable thermal diffusivity. Heat and Mass Transfer 41, 1048-1055.

Sharma, P.R. and R.N. Jat (1994). Flow and heat transfer between two vertical plates under viscous fluid injection through porous plate, the other being stretching sheet. Indian J. Technology, India XXXIII, 21-30.

Sharma, P.R and U. Mishra (2001). Steady MHD flow through horizontal channel: lower being a stretching sheet and upper being a permeable plate bounded by porous medium. Bull. Pure Appl. Sciences, India 20E, 175-181.

Sriramalu, A., N. Kishan, and R.J. Anand (2001). Steady flow and heat transfer of a viscous incompressible fluid flow through porous medium over a stretching sheet. J. of Energy, Heat and Mass Transfer 23, 483495.

Vajravelu, K. and J. Nayfeh (1993). Convective heat transfer at a stretching sheet. Acta Mechanica 96, 4754.

Watanabe, T. (1986). Magnetohydrodynamic stability of boundary layer along a flat plate with pressure gradient. Acta Mechanica 65, 41-50.

Watanabe, T. (1988). Effect of uniform suction or injection on a magneto-hydrodynamic boundary layer flow along a flat plate with pressure gradient. Acta Mechanica 73, 33-44. 
P. R. Sharma and G. Singh / JAFM, Vol. 2, No. 1, pp. 13-21, 2009.

Table 1 Values of $\mathrm{f}^{\prime \prime}(0)$ for different values of $\lambda$ are compared with the results obtained by Pop, Grosan and Pop (2004) and Mahapatra and Gupta (2002).

\begin{tabular}{|c|c|c|c|}
\hline \multirow{2}{*}{$\lambda$} & \multicolumn{3}{|c|}{$f^{\prime \prime}(0)$} \\
\cline { 2 - 4 } & $\begin{array}{c}\text { Pop, Grosan and Pop } \\
(2004)\end{array}$ & $\begin{array}{c}\text { Mahapatra and Gupta } \\
(2002)\end{array}$ & Present paper \\
\hline 0.1 & -0.9694 & -0.9694 & -0.969386 \\
\hline 0.2 & -0.9181 & -0.9181 & -0.9181069 \\
\hline 0.5 & -0.6673 & -0.6673 & -0.667263 \\
\hline 2.0 & 2.0174 & 2.0175 & 2.01749079 \\
\hline 3.0 & 4.7290 & 4.7293 & 4.72922695 \\
\hline
\end{tabular}

Table 2 Values of $\theta^{\prime}(0)$ for different values of $\varepsilon$ are compared with the results obtained by Chiam (1998).

\begin{tabular}{|c|c|c|}
\hline & & $-\theta^{\prime}(0)$ \\
\hline$\varepsilon$ & Chiam (1998) & Present paper \\
\hline 0.0 & 0.224886 & 0.22489 \\
\hline 0.05 & 0.214397 & 0.21440 \\
\hline 0.1 & 0.204844 & 0.20485 \\
\hline
\end{tabular}

Table 3 Values of $\theta^{\prime}(0)$ for different values of $\lambda$ are compared with the results obtained by Pop, Grosan and Pop (2004) and Mahapatra and Gupta (2002).

\begin{tabular}{|c|c|c|c|}
\hline \multirow{2}{*}{$\lambda$} & \multicolumn{3}{|c|}{$-\theta^{\prime}(0)$} \\
\cline { 2 - 4 } & Pop, Grosan and Pop (2004) & $\begin{array}{c}\text { Mahapatra and Gupta } \\
(2002)\end{array}$ & Present paper \\
\hline 0.1 & 0.081 & 0.081 & 0.081245 \\
\hline 0.5 & 0.135 & 0.136 & 0.135571 \\
\hline 2.0 & 0.241 & 0.241 & 0.241025 \\
\hline
\end{tabular}

Table 4 Values of $f^{\prime \prime}(0)$ for different values of $\lambda$ and $M$.

\begin{tabular}{|c|c|c|c|c|c|}
\hline \multirow{2}{*}{$\lambda$} & \multicolumn{5}{|c|}{$f^{\prime \prime}(0)$} \\
\cline { 2 - 5 } & $M=0.0$ & $M=0.1$ & $M=0.5$ & $M=1.0$ & $M=1.5$ \\
\hline 0.1 & $-0.969386\left(\eta_{\infty} \approx 10\right)$ & -0.97350851 & -1.067898 & -1.321111 & -1.66020742 \\
\hline 0.2 & $-0.9181069\left(\eta_{\infty} \approx 7\right)$ & -0.921466 & -1.00469 & -1.2156222 & -1.50849937 \\
\hline 0.5 & $-0.667263\left(\eta_{\infty} \approx 6\right)$ & -0.669102 & -0.71189085 & -0.83212508 & -1.00168146 \\
\hline 2.0 & $2.01749079\left(\eta_{\infty} \approx 4\right)$ & 2.01993243 & 2.07771118 & 2.2408579 & 2.50959738 \\
\hline 3.0 & $4.72922695\left(\eta_{\infty} \approx 3\right)$ & 4.73339915 & 4.8325013 & 5.13030441 & 5.59253315 \\
\hline
\end{tabular}


P. R. Sharma and G. Singh / JAFM, Vol. 2, No. 1, pp. 13-21, 2009.

Table 5 Values of rate of heat transfer for different values of $M, \lambda, \varepsilon, S$ and Pr.

\begin{tabular}{|c|c|c|c|c|c|}
\hline & & \multicolumn{2}{|c|}{$\begin{array}{l}M=0.0 \text { and } \lambda=0.1 \\
-\theta^{\prime}(0) \quad\left(\eta_{\infty} \approx 100\right)\end{array}$} & \multicolumn{2}{|c|}{$\begin{array}{c}M=0.5 \text { and } \lambda=0.1 \\
-\theta^{\prime}(0)\end{array}$} \\
\hline$\varepsilon$ & $S$ & $\mathrm{Pr}=0.01$ & $\operatorname{Pr}=0.023$ & $\operatorname{Pr}=0.01$ & $\operatorname{Pr}=0.023$ \\
\hline 0.0 & 0.0 & 0.030282 & 0.049828 & 0.029868 & 0.048889 \\
\hline 0.1 & 0.0 & 0.028235 & 0.046372 & 0.027859 & 0.045517 \\
\hline 0.0 & 0.1 & 0.007862 & 0.017913 & 0.007223 & 0.016482 \\
\hline 0.1 & 0.1 & 0.007172 & 0.016308 & 0.006570 & 0.015002 \\
\hline 0.0 & -0.1 & 0.044047 & 0.070101 & 0.043692 & 0.069310 \\
\hline \multirow[t]{2}{*}{0.1} & -0.1 & 0.041177 & 0.065452 & 0.043692 & 0.069310 \\
\hline & & \multicolumn{2}{|c|}{$\begin{array}{c}M=0.0 \text { and } \lambda=0.5 \\
-\theta^{\prime}(0) \quad\left(\eta_{\infty} \approx 75\right)\end{array}$} & \multicolumn{2}{|c|}{$\begin{array}{c}M=0.5 \text { and } \lambda=0.5 \\
-\theta^{\prime}(0)\end{array}$} \\
\hline$\varepsilon$ & $S$ & $\operatorname{Pr}=0.01$ & $\operatorname{Pr}=0.023$ & $\operatorname{Pr}=0.01$ & $\operatorname{Pr}=0.023$ \\
\hline 0.0 & 0.0 & 0.058418 & 0.090049 & 0.058323 & 0.089842 \\
\hline 0.1 & 0.0 & 0.054711 & 0.087048 & 0.054624 & 0.084109 \\
\hline 0.0 & 0.1 & 0.050281 & 0.077804 & 0.050180 & 0.077582 \\
\hline 0.1 & 0.1 & 0.047069 & 0.072796 & 0.046977 & 0.072594 \\
\hline 0.0 & -0.1 & 0.065803 & 0.101174 & 0.065712 & 0.100977 \\
\hline \multirow[t]{2}{*}{0.1} & -0.1 & 0.061647 & 0.094749 & 0.061564 & 0.094570 \\
\hline & & \multicolumn{2}{|c|}{$\begin{array}{c}M=0.0 \text { and } \lambda=2.0 \\
-\theta^{\prime}(0)\left(\eta_{\infty} \approx 30\right)\end{array}$} & \multicolumn{2}{|c|}{$\begin{array}{c}M=0.5 \text { and } \lambda=2.0 \\
-\theta^{\prime}(0)\end{array}$} \\
\hline$\varepsilon$ & $S$ & $\operatorname{Pr}=0.01$ & $\operatorname{Pr}=0.023$ & $\operatorname{Pr}=0.01$ & $\operatorname{Pr}=0.023$ \\
\hline 0.0 & 0.0 & 0.110397 & 0.165693 & 0.110445 & 0.165796 \\
\hline 0.1 & 0.0 & 0.103574 & 0.155495 & 0.103618 & 0.155590 \\
\hline 0.0 & 0.1 & 0.106408 & 0.159612 & 0.106456 & 0.159719 \\
\hline 0.1 & 0.1 & 0.099829 & 0.149789 & 0.099873 & 0.149886 \\
\hline 0.0 & -0.1 & 0.114288 & 0.171621 & 0.114336 & 0.171723 \\
\hline 0.1 & -0.1 & 0.107227 & 0.161059 & 0.107270 & 0.161152 \\
\hline
\end{tabular}

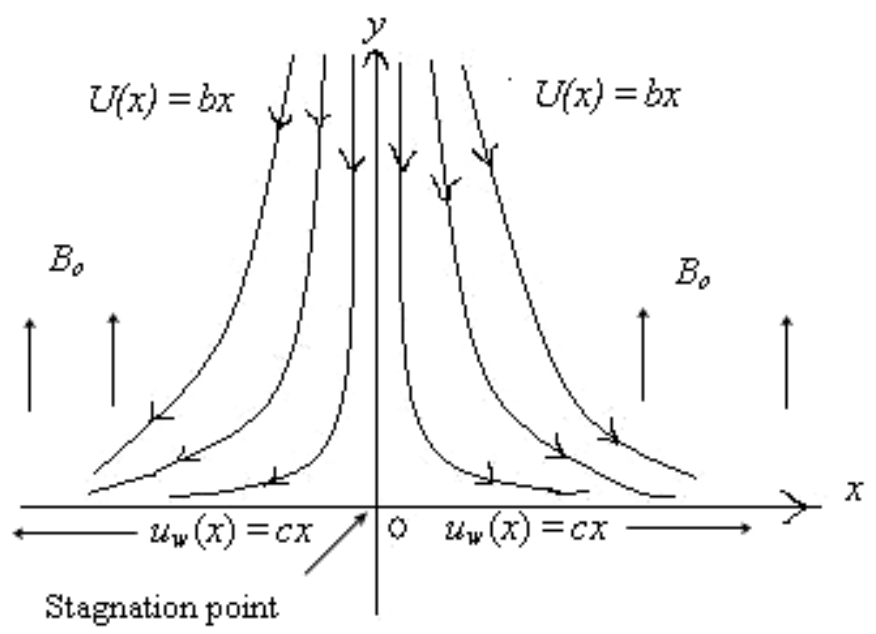

Fig. 1. Physical model 
P. R. Sharma and G. Singh / JAFM, Vol. 2, No. 1, pp. 13-21, 2009.

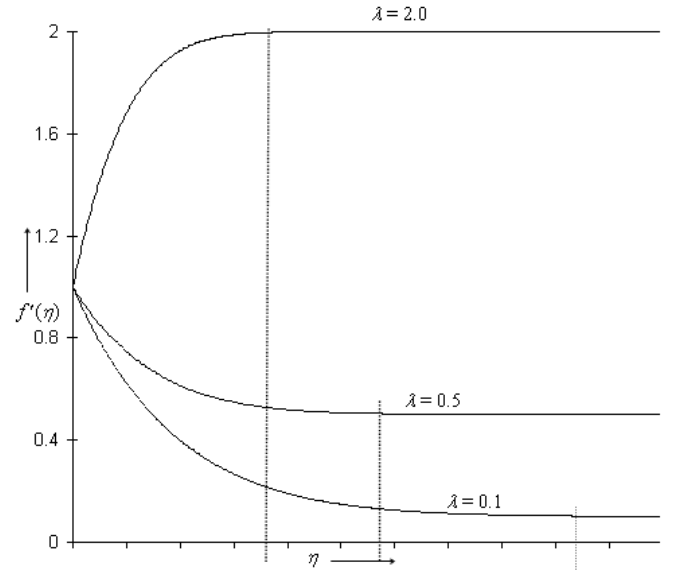

Fig. 2. Velocity distribution versus $\eta$ when $M=0.0$.

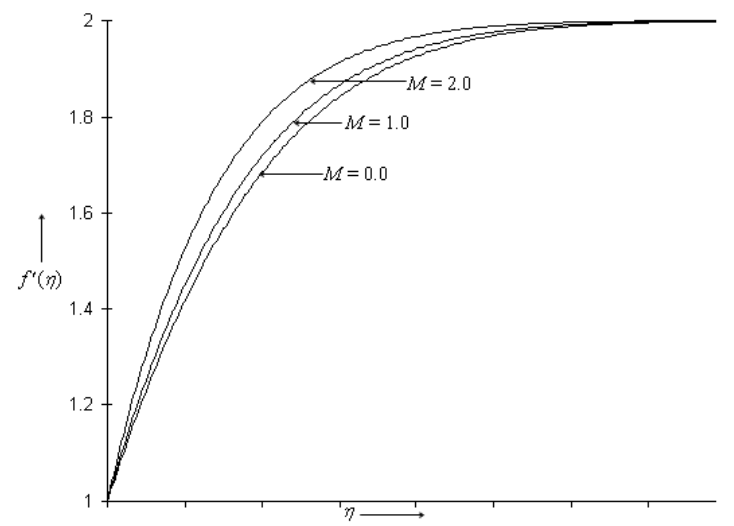

Fig. 4. Velocity distribution versus $\eta$ when $\lambda=2.0$.

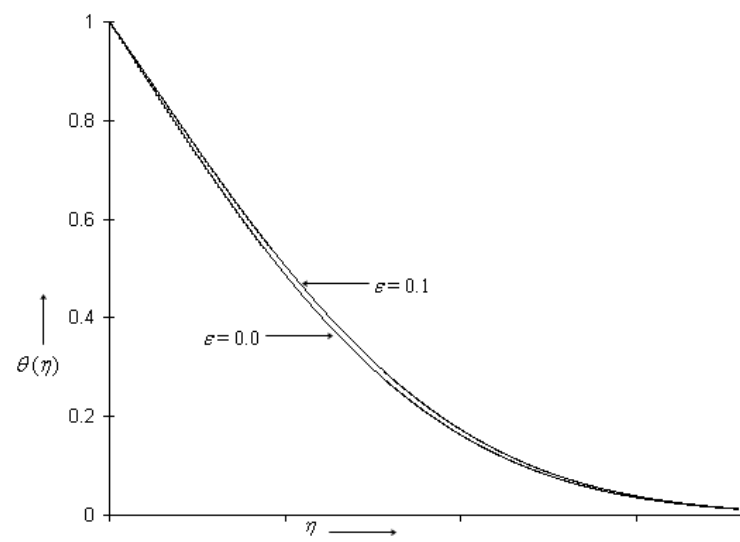

Fig. 6. Temperature distribution versus $\eta$ when $M=0.0$, $\lambda=2.0, S=0.0$ and $\operatorname{Pr}=0.01$.

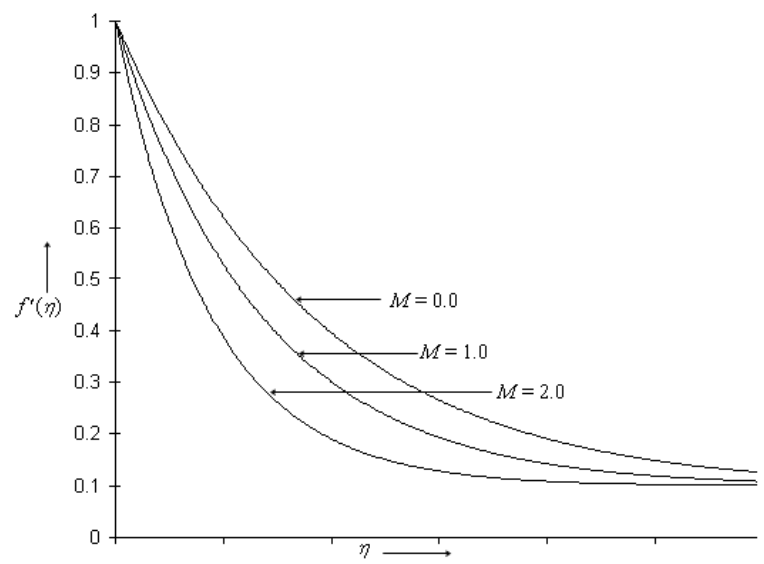

Fig. 3. Velocity distribution versus $\eta$ when $\lambda=0.1$.

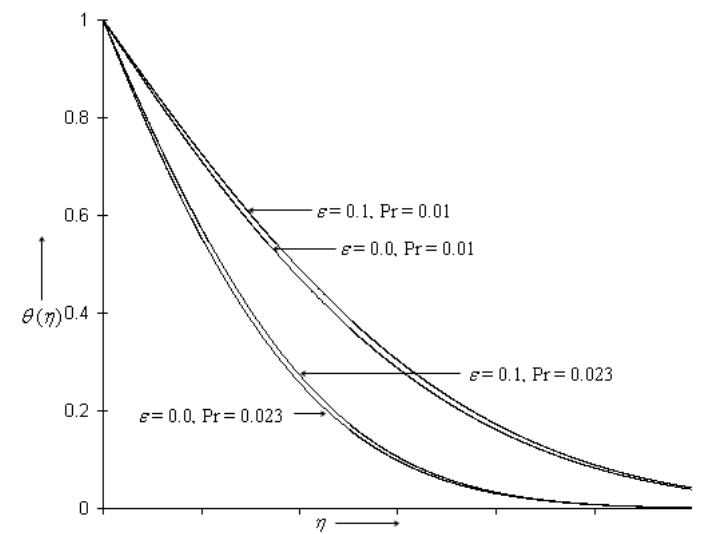

Fig. 5. Temperature distribution versus $\eta$ when $M=0.0$, $\lambda=0.1$ and $S=0.0$.

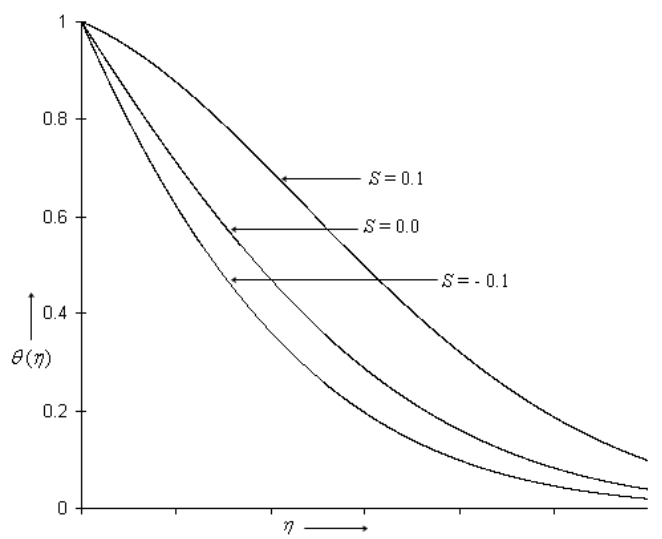

Fig. 7. Temperature distribution versus $\eta$ when $M=0.0$, $\lambda=0.1, \varepsilon=0.0$ and $\operatorname{Pr}=0.01$. 
P. R. Sharma and G. Singh / JAFM, Vol. 2, No. 1, pp. 13-21, 2009.

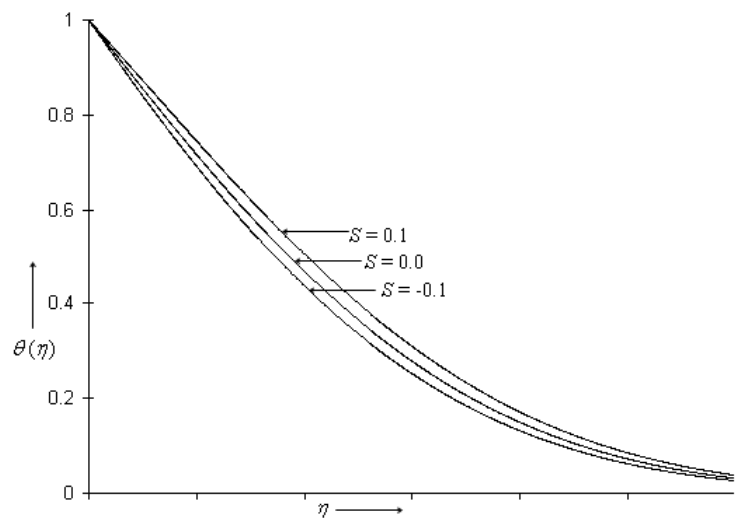

Fig. 8. Temperature distribution versus $\eta$ when $M=0.0$, $\lambda=0.5 \varepsilon=0.0$ and $\operatorname{Pr}=0.01$.

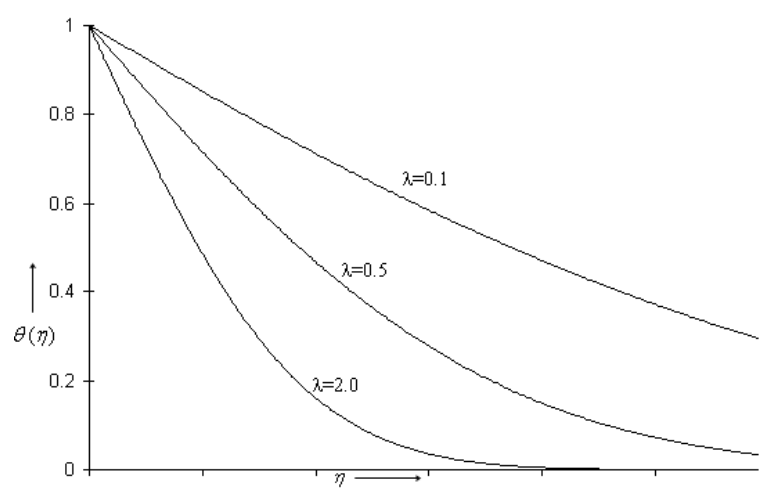

Fig. 10. Temperature distribution versus $\eta$ when $M=0.0, S=$ $0.0, \varepsilon=0.0$ and $\operatorname{Pr}=0.01$.

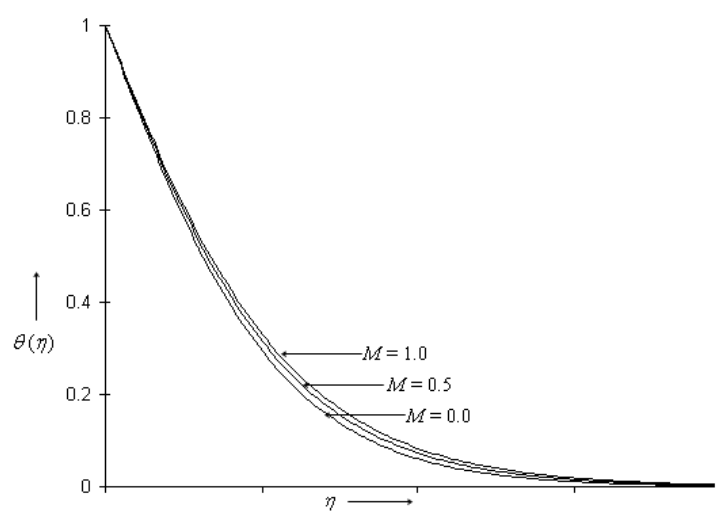

Fig. 12. Temperature distribution versus $\eta$ when $\lambda=0.5$, $\varepsilon=0.0, S=0.1$ and $\operatorname{Pr}=0.023$.

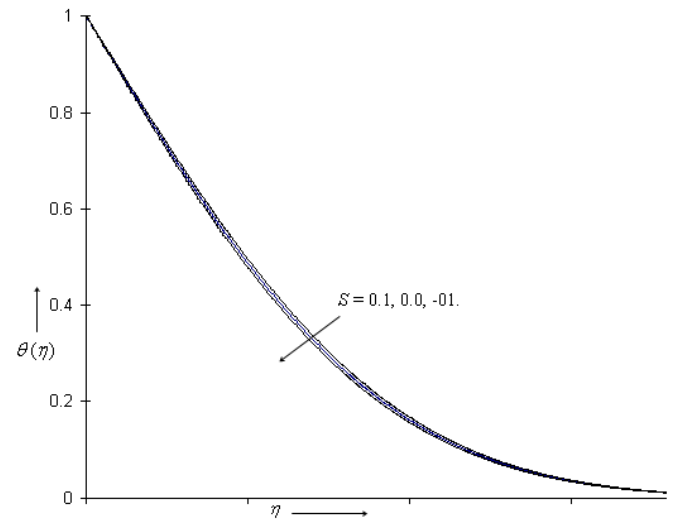

Fig. 9. Temperature distribution versus $\eta$ when $M=0.0$, $\lambda=2.0, \varepsilon=0.0$ and $\operatorname{Pr}=0.01$.

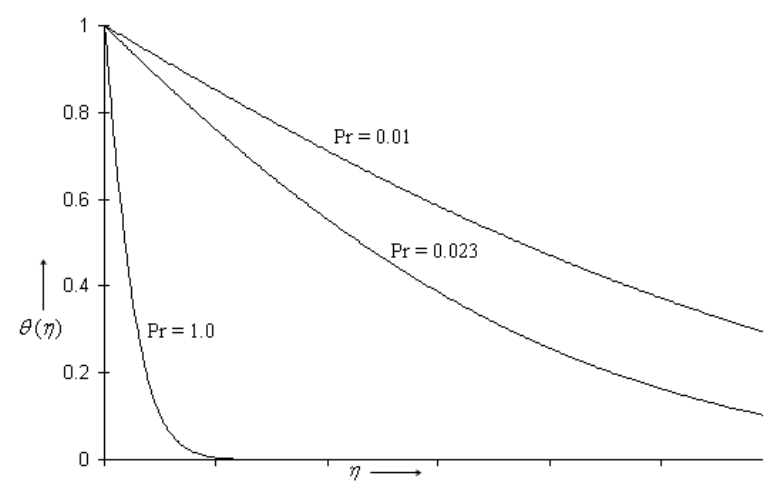

Fig. 11. Temperature distribution versus $\eta$ when $M=0.0, \quad S$ $=0.0, \varepsilon=0.0$ and $\lambda=0.1$. 